\title{
THE SYNTHESIS AND PHOTOSENSITIVITY OF ELEOSTEARIC ACID-RESORCIN SERIES PHENOLIC RESINS
}

\author{
Wang Liyuan and Yu Shangxian \\ Institute of Applied Chemistry, Beijing Normal University, Beijing 100875, China
}

\begin{abstract}
A series of eleostearic acid-resorcin phenolic resins were synthesized, mainly including eleostearic acid-resorcin-formaldehyde resin ( I )(softening point: $70-75^{\circ} \mathrm{C}$ ), methyl eleostearate-resorcin-formaldehyde resin( II )(softening point: $65-70^{\circ} \mathrm{C}$ ), $\mathrm{N}$ - hydroxyethyl eleostearic amide - resorcin - formaldehyde(III)(softening point: $85-90^{\circ} \mathrm{C}$ ). The relative molecular weights determined by GPC method are $\overline{\mathrm{M}}_{n}: 5.0 \times 10^{3}-1.0 \times 10^{4}, \overline{\mathrm{M}}_{w}: 1.0$ $\times 10^{4}-4.0 \times 10^{4}, \mathrm{D}: 3.0-6.0$. The reaction products were characterized using I.R.and elemental analysis. A detailed investigation was made on the photosensitivity of the photopolymers formed from these phenolic resins and $\mathrm{BAC}-\mathrm{M}$. All these negative photoresists can be developed in dilute aqueous base. The minimum quantities of exposure energy required are between $10-20 \mathrm{mj} / \mathrm{cm}^{2}$. Clear etched image with $3 \mu \mathrm{m}$ line width was obtained. The photocured films have excellent resistance to acids.
\end{abstract}

\section{Introduction}

Our laboratory had succeded in synthesizing tung oil-resorcin resin, studied and reported on the photosensitivity of the photopolymer made of tung oil-resorcin and $\mathrm{BAC}-\mathrm{M}[1]$ and made a detailed investigation into the photocrosslinking mechanism [2]. In order to further increase the softening point and improve the performance of the photosensitive film, so that photoresist of some practical value 
could be obtained, in this paper we synthesized the phenolic resins. First, to start at the reaction of eleostearic acid and resorcin, the additive products are obtained with the structure shown as formula (1):

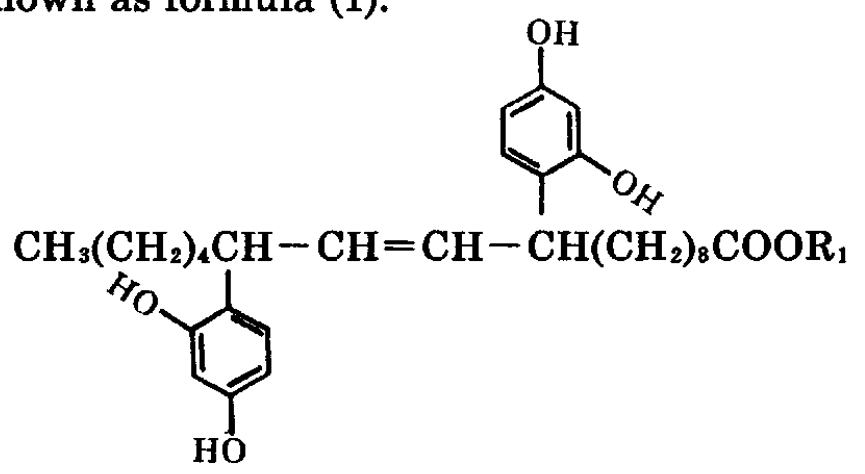

where $\mathrm{R}_{1}: \mathrm{H}, \mathrm{CH}_{3}$. These additive products of resorcin continue to react with formaldehyde to form linear eleostearic acid-resocin-formaldehyde(EARF)( I )and methyl eleostearate-resorcin-formaldehyde(MERF)(II). The aminolysis reaction of thus obtained MERF and ethanolamine gives $\mathrm{N}$-hydroxyethyl eleostearic amide - resorcin-formaldehyde(NEARF)(III). In these phenolic resins, since one or two reactive sites are retained on every resorcin and no less than one double bond remains unreacted on every eleostearic acid, they can form negative photoresists together with diazids and polyhalides. This paper reports in the main the synthesis, characterization of these phenolic resins and the photosensitivity of the photoresists made of these resins and $\mathrm{BAC}-\mathrm{M}$

\section{Experimental}

\subsection{Synthesis}

Eleostearic acid is obtained by the method [3] of saponifying and then acidifying tung oil which is available commercially. The eleostearic acid reacts with twice the moles of resorcin for several hours at $100-130^{\circ} \mathrm{C}$ under the catalysis of acids and nitrogen atomosphere, the obtained additive product is shown as $(1)\left(R_{1}=H\right)$. The product, washed several times with hot water to remove the unreacted resorcin, is a brown sticky substance, which is soluble in dilute aqueous base. In this product are mixed some fatty acids to which resorcin is not inducted [3].

In four times the moles of methanol, crude eleostearic acid is heated and refluxed with the catalysis of concentrated sulfuric acid or $p$-methyl phenyl sulfonic acid and gives a esterified product, the main part of which is methyl eleostearate(ME). This product, after purified by water washing, reacts with resorcin, the additive product is also shown as (1).

Eleostearic acid and methyl eleostearate are mixed in a specific proportion and 
react with resorcin and then a mixture of eleostearic acid-resorcin (EAR) and methyl eleostearate-resorcin (MER) is obtained.

The condensation reaction of the above obtained additive products of resorcin and formalin at the presence of acid catalysts gives phenolic resins EARF (I), MERF (II) and a copolymer of eleostearic acid and methyl eleostearate.

The aminolysis reaction of the above obtained MERF (II) and ethanolamine at $140-160^{\circ} \mathrm{C}$ gives NEARF (III).

\subsection{Determination of photosensitivity}

2,6-Bis-(azidobenzal)-4-methyl-cyclohexanone $(\mathrm{BAC}-\mathrm{M})$ is added at an amount of 5wt.\% of resin to the ethylene glycol ethyl ether solution of about 10 wt. \% of phenolic resin and then a solution of photopolymer used for determination is obtained. This photosensitive solution is coated on the aluminum base used for PS plate, or diluted to a solution of about $5 \mathrm{wt} . \%$ and coated on aluminum film, and then the experiments of exposure, development, etching and resistance are performed.

\section{Results and Discussions}

\subsection{Characterization of reaction products}

All the reaction processes can be surveyed by infrared spectrum of the products. After the eleostearic acid is esterified, the carbonyl infrared absorption shifts from about $1705 \mathrm{~cm}^{-1}$ to about $1735 \mathrm{~cm}^{-1}$. The additive products of resorcin have strong phenolic hydroxyl infrared absorption around $3350 \mathrm{~cm}^{-1}$, and the absorprion of conjugated double bonds around $2980 \mathrm{~cm}^{-1}$ mostly dissappears. NEARF resin has carbonyl absorption around $1650 \mathrm{~cm}^{-1}$. Figure 1 gives the I.R. spectrum of methyl eleostearate-resorcin.

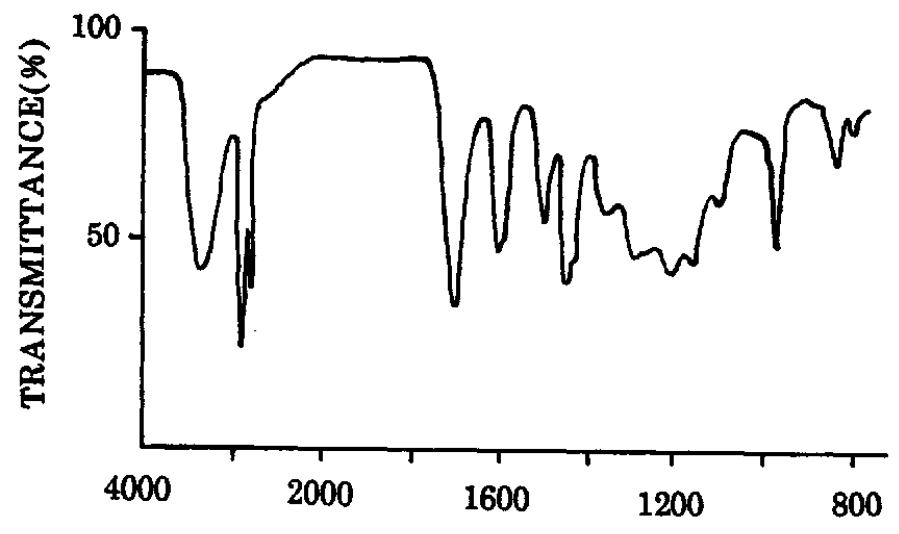

WAVENUMBER $\mathrm{CM}^{-1}$

Figure 1 IR Spectrum of methyl eleostearate-resorcin

Some results of elemental analysis are listed in Table 1, from which, the inducting rates of resorcin to eleostearic acid and methyl eleostearate can be 
estimated.

Table 1 Elemental Analysis of Some Products

\begin{tabular}{cccc}
\hline ME & EAR & MER & NEARF \\
\hline C:77.10 & C:73.29 & C:73.26 & C:72.29 \\
H:11.75 & H:9.61 & H:9.41 & H:9.30 \\
O:11.15 & $0: 17.10$ & $0: 17.33$ & $0: 15.51$ \\
& & & N:2.91 \\
\hline
\end{tabular}

The results show that about 1.2 moles of resorcin was inducted to one mole of eleostearic acid and that about $\mathbf{1 . 5}$ moles of resorcin was inducted to one mole of methyl eleostearate. EAR product contains a small amount of unreacted fatty acid, thus lowering the inducting rate. In MER product, the unreacted methyl esters can be evaporated above $190^{\circ} \mathrm{C}$ at high vacuum, or they can be extracted using petroleum ether after the resorcin additive product is resolved by aqueous base. The removed substance is about $10-14 \mathrm{wt} . \%$ of the total amount.

In the process of formaldehyde reaction, crosslinking may take place, since there exist two reactive sites on every resorcin. In our experiment it was found that crosslinking would be easily resulted when the formalin added was superfluous and the reaction temperature was too high. So the suitable amount of formalin and reaction temperature must be maintained. Some properties of the phenolic resins are summarized in Table 2.

Table 2 Characteristics of the Phenolic Resins

\begin{tabular}{cccccccc}
\hline No. & appearance & softening & Viscosity* & \multicolumn{4}{c}{ solubility } \\
& & point $\left({ }^{\circ} \mathrm{C}\right)$ & (cp) & aceton & dioxane & ethanol & DMF \\
\hline (I) & brown & $70-75$ & 7.23 & soluble & soluble & soluble & soluble \\
(II) & brown & $65-70$ & 6.18 & $\ldots$ & $\ldots$ & $\ldots$ & $\ldots$ \\
(III) & brown & $85-90$ & 6.20 & $\ldots$ & $\ldots$ & $\ldots$ & $\ldots$ \\
\hline
\end{tabular}

* at $20^{\circ} \mathrm{C}, 10 \mathrm{wt} . \%$ resin(ethylene glycol ethyl ether as solvent)

The relative molecular weights of the phenolic resins were determined by GPC. The result is $\overline{\mathrm{M}} \mathrm{n}: 5.0 \times 10^{3}-1.0 \times 10^{4}, \overline{\mathrm{M}}_{\mathrm{w}}: 1.0 \times 10^{4}-4.0 \times 10^{4}, \mathrm{D}: 3.0-6.0$. Figure 2 gives the results of resin (I )and (II). Here, the unreacted methyl ester was removed from resin (II). The size of molecular weight is related to the amount of formalin added and reaction temperature, when the two factors are determined, the molecular weight will increase with reaction time. 


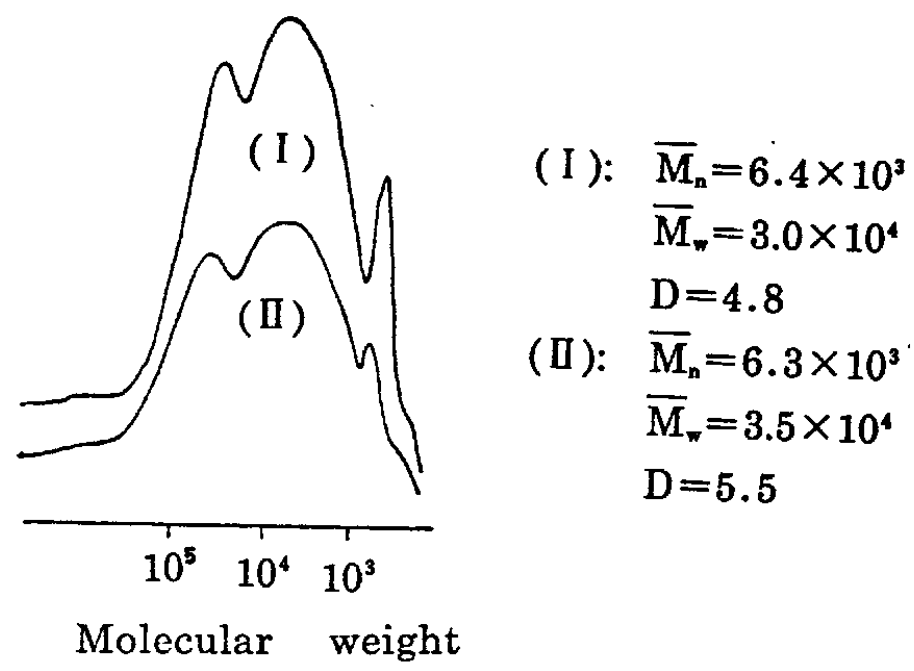

Figure 2 The molecular weight distribution of resin ( I ) and (II) by GPC

\subsection{Investigation on photosensitivity}

\subsubsection{Photosensitivity determination}

The photosensitive solution was spin coated on the aluminum base and, after baked at $70^{\circ} \mathrm{C}$ for 3 minutes, the photopolymer film was exposed under the cover of Kodark Photographic Step Tablet No. 2, the number of residual segments on the tablet was observed after developed with aqueous base to obtain the relative photosensitivity. The exposure power is $3.0 \mathrm{mw} / \mathrm{cm}^{2}$. The data of the photopolymer made of resin (II) are given in Table 3. The minimum quantity of exposure energy required of photopolymer (II) can be calculated from the formula $E=I_{0} \mathrm{Tt}$ and the data in Table 3 to be about $15 \mathrm{~mJ} / \mathrm{cm}^{2}$. The measured Values of Emin for these photopolymers are in the range of $10-20 \mathrm{~mJ} / \mathrm{cm}^{2}$.

Table 3 The Relative Photosensitivity of Photopolymer(II)

\begin{tabular}{ccccc}
\hline exposure time(min) & 0.5 & 1.0 & 1.5 & 2.0 \\
residual segments & $5-6$ & $7-8$ & $8-10$ & $9-10$ \\
\hline
\end{tabular}

\subsubsection{Resolution and resistance to etchants}

The photopolymers were coated on thin Aluminum film and baked with a resist thickness of $2 \mu \mathrm{m}$. After exposed and developed, the film was etched in concentrated phosphoric acid at $40^{\circ} \mathrm{C}$ and the clear etched image with $2 \mu \mathrm{m}$ line width was obtained. The photocured resists have excellent resistance to chlorhydric acid, sulphuric acid, dilute nitric acid and fluorine hydride. To meet the requirement of photopolymers for practical uses, the solubilities of some commonly used solvents on the photocured film were also measured. Some experimental results are 
summarized in Table 4.

\subsubsection{Performance of development}

All the photopolymers can be developed with dilute aqueous base. Some developing properties of the photopolymers are summarized in Table 5. It was found that the base resistance of photopolymer( I ) is very weak and that of photopolymer (II) is too strong to be developed with pure aqueous base. So, it is quite difficult for (I) and (II) to find suitable developer to obtain the best developing effect. The developing condition for (III) can be easily deteremined and very good developing effect has been obtained. But the brightness and lipotropism of photocured film of (II) are the best among these three kinds of photopolymers. In order to find a suitable developer and the best developing effect, the copolymer of eleostearic acid and methyl eleostearate was synthesized.

Table 4 The Resistance of Photocured Films to some Acids and Solvents

\begin{tabular}{|c|c|c|c|c|c|}
\hline acids and solvents & $85 \% \mathrm{H}_{3} \mathrm{PO}_{4}$ & $1 \% \mathrm{HCl}$ & Water & $\begin{array}{l}\text { dimethyl } \\
\text { benzene }\end{array}$ & kerosene \\
\hline temperature $\left({ }^{\circ} \mathrm{C}\right)$ & 40 & 15 & $10-15$ & $10-15$ & $10-15$ \\
\hline time(hour) & 1.0 & 2.5 & 24 & 12 & 12 \\
\hline rate of $\quad$ (I) & $91 \%$ & $94 \%$ & $100 \%$ & $90 \%$ & $100 \%$ \\
\hline residual film (II) & $96 \%$ & $98 \%$ & $100 \%$ & $70 \%$ & $100 \%$ \\
\hline
\end{tabular}

Table 5 Developing Properties of the Photopolymers

\begin{tabular}{cccc}
\hline No. & (I) & (II) & (II) \\
\hline developer & $2.5 \%$ & $8 \% \mathrm{Na}_{2} \mathrm{SiO}_{3}$ & $5 \% \mathrm{Na}_{2} \mathrm{SiO}_{3}$ \\
& $\mathrm{HOC}_{2} \mathrm{H}_{4} \mathrm{NH}_{2}$ & $3 \%$ organic solvent & \\
& & $1 \%$ surfactant & \\
developing time(min) & $0.5-1.0$ & $1.0-1.5$ & $1.0-1.5$ \\
rate of residual film(5mins) & $90 \%$ & $92 \%$ & $96 \%$ \\
\hline
\end{tabular}

exposure time:1.5min developing temperature:20-25 $\mathrm{C}$

Table 6 Developing Properties of Photopolymers of partly esterified EARF Resin

\begin{tabular}{cccc}
\hline ester(\%)percentage & 20 & 30 & 40 \\
developer & $1.5 \% \mathrm{Na}_{3} \mathrm{PO}_{4}$ & $4 \% \mathrm{Na}_{2} \mathrm{SiO}_{3}$ & $4 \% \mathrm{Na}_{2} \mathrm{SiO}_{3}$ \\
& & & $4 \%$ NaOH \\
& & & 2 surfactant \\
developing time $(\min )$ & 0.5 & $1.0-1.5$ & $1.0-1.5$ \\
\hline
\end{tabular}
exposure time: $1.5 \mathrm{mins}$, developing temperature:20-25 $\mathrm{C}$ 
The developing performance of the photopolymers made of thus obtained partly esterified EARF resin is shown in Table 6. In our experiments, the developing effect is very satisfactory when the esterification percentage is about $30 \%$.

Acknoledgment-We would thank Huang Lan, Chemistry Department of Beijing University, for her assistance in taking the GPC measurements.

\section{References}

1 Yu shangxian, et al, J.Photopolym. Sci. Technol, 1988, 1(2):171

2 Yu shangxian, et al, J.Beijing Normal University (Natural science), 1990,No.4, PP.68-73

3 Ren Zhi, Tung Oil Synthesized Rubber, Shanghai(1959);P8. 